\title{
STELLAR DATING AND FORMATION \\ OF GALACTIC SPHEROIDS
}

\author{
ALVIO RENZINI \\ Dipartimento di Astronomia \\ CP 596, I-40100 Bologna, Italy \\ (astbo3::alvio@alma02.bo.astro.it)
}

\section{Introduction}

In recent years a view spread widely according to which spheroidal stellar systems are not as old as argued by Baade forty years ago and for a long time given for granted. In this - more modern view - most galactic bulges may form late, e.g. as a spontaneous corruption of disks, and most ellipticals may seemingly be late comers, perhaps the result of merging spirals.

My task here is to play the devil's advocate, and argue that Baade was perhaps $\sim 90 \%$ right, after all, a conservative view with which I am sympathetic. Here a set of evidences are presented that appear to support the ancient picture according to which most of the stars in galactic bulges and ellipticals - the spheroidal populations for short - were produced during a relatively short, early phase of intense star formation, probably at redshifts beyond $\sim 2$. These facts deserve to be fully appreciated before declaring closed the quest for the age and formation processes of galactic spheroids.

\section{Age and Helium Estimates in the Galactic Bulge}

Sitting at the center of both the spheroid and the disk, it is no surprize if somebody relates the formation of the bulge to that of the halo, somebodyelse to that of the disk and its evolution. Is the bulge the core of the halo, and its formation is coeval with it? Or, is the bulge the inner part of the disk, and its formation a rather late event in the evolution of the Milky Way? In turn, the first option comes in two opposite versions. In one view star formation starts out in the halo, and progressively metal-enriched gas drips towards the center so that the innermost part of the spheroid - the 
bulge - forms last as the culmination of the condensation process (Larson, 1975). In the other view star formation first erupts in the central, high density regions of the protogalaxy. There it proceeds at a very high rate, locally rapidly enriching the gas to super metal rich abundances, until the heating of the interstellar medium discontinues further star formation (Renzini and Greggio, 1990; Larson, 1990; Lee, 1992; Renzini, 1993). Thus, in one view the bulge is on average slightly younger than the halo, slightly older in the other view.

A decisive step towards distinguishing among these competing scenarios should come from dating stars in the bulge. Recent studies of bulge fields all agree that the bulk of stars in the bulge are very old, older than at least 5-8 Gyr, but diverge on whether the data require the presence of a main component substantially younger than 15 Gyr (e.g., Terndrup, 1988; Renzini, 1993; Holtzman et al. , 1993). Paczyński et al. (1994) have recently drawn attention on the remarkable lack of disk stars towards Baade's Window, beyond the spiral arm at $2 \mathrm{kpc}$ from us. The CMD in their Fig. 1 also shows that very few stars lie close to the expected location of intermediate age MS for stars at the distance of the Galactic center. This indicates that indeed star formation in the bulge has ceased at least several Gyr ago, though the CMD is not deep enough for a precise dating using the MS turnoff. Paczyński et al. notice instead the exceptionally high ratio of the number of red clump to RGB stars $(\sim 4 / 3$, see their paper for details), and on this ground suggest an intermediate age for the bulk of stars in the bulge. The data allow however a different interpretation (Renzini, 1994a), as the clump to RGB ratio is actually very insensitive to age while it shows a strong dependence on composition, i.e., on both $Z$ and $Y$ (Iben, 1968). This follows from the composition dependence of the clump and RGB lifetimes, and - working differentially with respect to the globular cluster M3 - a clump to RGB ratio $4 / 3$ is reproduced with $Y=0.35$ for an assumed average metallicity of bulge stars $Z=0.04$. This is in substantial agreement with an earlier estimate by Terndrup (1988), based on a somewhat different version of the same method, and gives a helium enrichment parameter $\Delta Y / \Delta Z \simeq 3$ for bulge stars. One may argue that the average metallicity of bulge stars is more likely just solar (McWilliam and Rich, 1994); moreover, direct retrival of Paczyński et al. database reveals a clump to RGB ratio $=$ $32970 / 28146=1.17$, rather than $4 / 3$. Both points favor a lower $Y$, but a somewhat higher $\Delta Y / \Delta Z$. Though the helium abundance has been rarely regarded as an important parameter in extragalactic stellar population studies, its relevance will be put into focus in $\S 4$.

Precise age dating of bulge stars is hampered by several factors, including crowding, variable reddening, metallicity dispersion, different distances along the line of sight due to the finite depth of the bulge, and contami- 


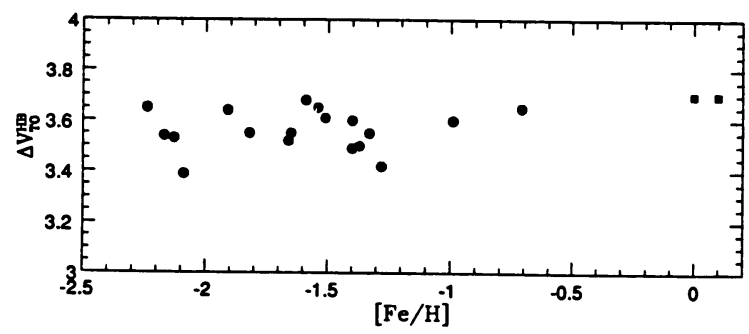

Figure 1. The HB to MS turnof$\mathrm{f}$ luminosity difference as a function of metallicity for a representative set of galactic globular clusters. The two bulge clusters NGC 6528 and NGC 6553 studied with HST/WFPC2 are the most metal rich ones. For them I have adopted here $[\mathrm{Fe} / \mathrm{H}]=0.0$ and +0.1 , respectively.

nation by foreground disk stars. Some of these limitations can be avoided by studying globular star clusters that are physically located in the bulge, and whose high metallicity ensures that are not metal poor halo interlopers presently close to their galactic pericenter. In this perspective the globular clusters NGC 6528 and NGC 6553 were selected for HST imaging with WFPC2 (Ortolani et al., 1994). From the resulting CMDs one can estimate a horizontal branch to turnoff luminosity difference $\Delta V_{\mathrm{TO}}^{\mathrm{HB}}=3.7 \pm 0.2$ for both clusters, a better accuracy being hampered by the strong differential reddening across the FoV. Fig. 1 shows $\Delta V_{\mathrm{TO}}^{\mathrm{HB}}$ vs. [Fe/H] for a representative sample of well populated galactic globular clusters: it appears that the flat run of this well known age indicator with metallicity continues al1 the way to the two very metal rich clusters in the bulge, i.e., halo and bulge clusters do not appear to belong to radically distinct populations, with (very) different star formation epochs.

In summary, two main conclusions emerge from Ortolani et al. study: 1) the (super) metal rich globulars in Baade's Window appear to be as old as halo globulars (within the errors!), and 2) the bulge was enriched to metallicities near or above solar in less (perhaps much less!) than a few Gyr. Admittedly errors are still large $(\sim 20 \%$ in relative age compared to halo clusters), and do not allow to distinguish between the inside-out and the outside-in options schetched above. Still these results appear to favor an early and fast formation of the bulge, over a slow and/or late formation process. The apparent age continuity between halo and bulge globulars also argues for the bulge formation being just part of the spheroid formation process, rather than a late event in the evolution of the Galaxy.

\section{Nuclear Populations in Galactic Spheroids}

The SED of ellipticals and bulges is characterized by the typical UV upturn, extensively discussed by Ferguson at this meeting. Ongoing massive star formation was at some stage entertained as a possible origin of the UV upturn, but now an old star origin is more widely favored. It is worth recalling that estimates of the helium enrichment parameter - such as that 
reported in $\S 2-$ are of great value in this connection, as $\Delta Y / \Delta Z$ is one of the key factors controlling the ability of old stellar population to produce hot stars (Greggio and Renzini, 1990).

HST imaging in the UV for a sample of three galaxies was secured during 1993, with the aim of checking for a possible starburst contribution to the UV upturn (see poster by Bertola et al. in these proceedings). The UV surface brightness of the three galaxies appears to be as smooth as silk, with no clumps or knots that could be attributed to recent episodes of star formation. This lend further support to an old star origin of the UV upturn. There are however two notable exceptions: unresolved, UV bright central spikes are present in the galaxies NGC 4552 and NGC 2681. With an estimated luminosity of $\sim 10^{7} L_{\odot}$ their contribution to the UV flux detected by IUE is negligible, but they signal that something special is going on at the center of these galaxies. Non-stellar, weak AGN-like phenomena is a possibility, but existing evidence is also very reminiscent of what is going on at the center of our own Galaxy, where an extremely dense cluster of hot stars is present (Genzel et al., 1994), with comparable luminosity to that of the spikes in the two other galaxies.

There is now growing evidence that a nuclear stellar population with special characteristics may not be an uncommon phenomenon in galaxies (see also Crane et al. , 1993). As discussed by Genzel et al. for our Galaxy, a central cluster of hot stars may be the result of either occasional, highly confined bursts of star formation, or of stellar stripping and/or coalescence in an extremely high-density environment, when the star density exceeds $\sim 10^{7} M_{\odot} \mathrm{pc}^{-3}$. Starbursts, superdense clusters, and central black holes may not be mutually exclusive phenomena, at least from an evolutionary point of view. Indeed, repeated nuclear bursts of star formation may lead to the accumulation of a denser and denser cluster, until its dynamical evolution takes over leading to the formation and growth of a central black hole. It appears that the study of these UV bright central spikes may soon shed light on a variety of evolutionary phenomena in galactic nuclei.

\section{The $M / L$ of Stellar Populations and the Fundamental Plane}

Elliptical galaxies do not populate randomly the parameter space with coordinates central velocity dispersion $\sigma$, effective radius $R_{\mathrm{e}}$, and mean effective surface brightness $I_{\mathrm{e}}$. They are rather confined with very small dispersion about a plane (Dressler et al. , 1987; Djorgovski and Davis, 1987), thus called the fundamental plane (FP). Bender et al. (1992, BBF) have introduced an orthogonal coordinate system $\left(\kappa_{1}, \kappa_{2}, \kappa_{3}\right)$, each of the new variables being a linear combination of $\log \sigma^{2}, \log R_{\mathrm{e}}$, and $\log I_{\mathrm{e}}$. In the new space, the $\kappa_{1}-\kappa_{3}$ plane provides an almost edge-on view of the FP, with 
$\kappa_{1} \propto M$ and $\kappa_{3} \propto M / L$. For the BBF sample of Virgo and Coma cluster ellipticals the FP is tilted, with $\kappa_{3}$ increasing by $\Delta \kappa_{3} \simeq 0.3$ from faint to bright galaxies. From BBF one also has $\log M / L_{\mathrm{V}} \simeq-0.7+\sqrt{3} \kappa_{3}$, hence the tilt of the FP implies the trend $M / L_{\mathrm{V}} \propto L^{1 / 5} \propto M^{1 / 6}$ (see also Dressler et al. , 1987). The origin of the tilt has been left unexplored for a rather long time, perhaps because $\mathrm{a} \sim 1 / 5$ exponent is rather small. However, ellipticals in the BBF sample span a range $\sim 100$ in luminosity (mass), and therefore the observed tilt implies an increase by a factor $\sim 3$ in $M / L_{\mathrm{V}}$ along the FP. As quantitatively explored elsewhere (Renzini and Ciotti, 1993, RC), this may correspond to either a drastic variation of the stellar IMF along the FP, or of the dark matter content/distribution. In one case $\mathrm{M} /$ brown dwarfs should turn from a being a minor constituent, to dominate the baryonic mass of ellipticals. In the other, dark matter should turn from having very little influence within $\sim 1 R_{\mathrm{e}}$, to fully dominate the dynamics even in the central regions. There may well be a macroscopic variation in some crucial component of ellipticals along the FP, and we need to identify what it is.

A contribution to the tilt certainly comes from metallicity increasing along the FP, a consequence of the $M g_{2}-\sigma$ relation. Indeed, in more metal rich population one expects a shift to the red of the SED, thus depressing the optical luminosity. But the effect was estimated to be marginal (Dressler et al. , 1987), and RC have neglected it on the ground that the tilt appears to be present also when using the $K$-band luminosity (Djorgovski and Santiago, 1993). However, the metallicity dependence of the $M / L$ ratio was perhaps too prematurely dismissed, and I will now explore this aspect in some detail.

Figure 3 shows the $M / L_{\mathrm{V}}$ ratio vs [Fe/H] for a sample of galactic globular clusters and Virgo elliptical galaxies. There are three features to be noted: 1) globular clusters span over 2 dex in $[\mathrm{Fe} / \mathrm{H}]$, with no appreciable trend in $M / L ; 2)$ in contrast, elliptical galaxies show an extremely steep correlation of $M / L$ with $[\mathrm{Fe} / \mathrm{H}] ; 3)$ the most metal poor ellipticals have $M / L$ ratios comparable to that of the globulars. The two families of objects join with continuity in the diagram, yet at the junction there is the sharp change in slope between clusters and ellipticals. This sharp break has two possible interpretations. One is that the $M / L$ ratio of stellar populations has indeed very little direct dependence on metallicity, and the effect is due to dark matter - either barionic or not - playing a crucial role in galaxies while being inessential for globulars. Alternatively, the break could be a mere metallicity effect, after all. The first option was explored by CR; here I deal with the second.

Superimposed to the data points, Fig. 2 also shows the $M / L_{\mathrm{V}}$ ratio of the 15 Gyr simple population models of Buzzoni $(1989,1994)$. With a 


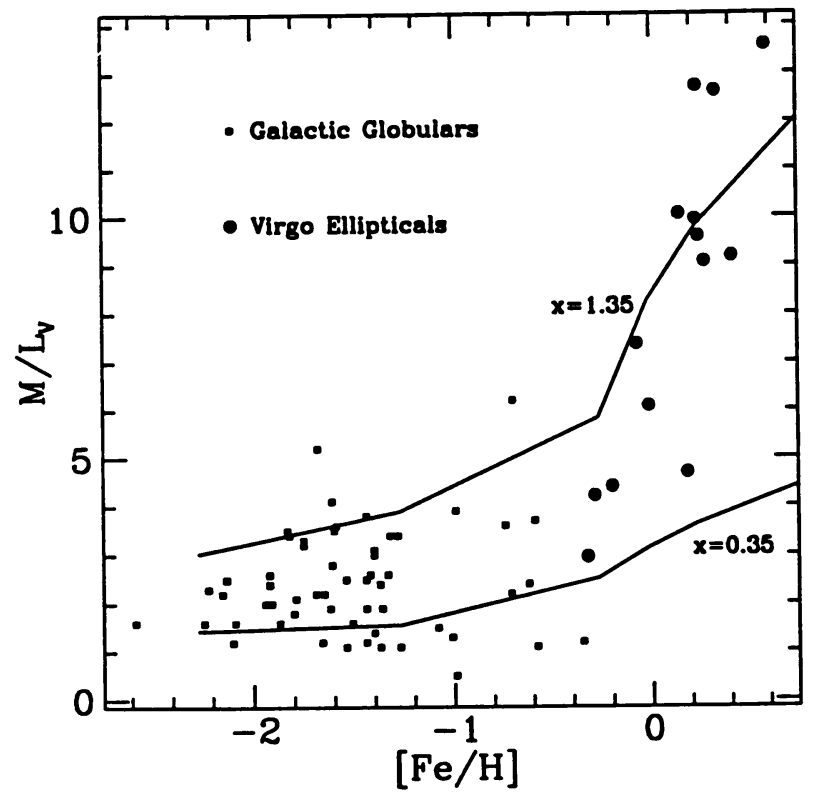

Figure 2. The mass to light ratios of Galactic globular clusters (from Pryor and Meylan, 1993) and of Virgo ellipticals (from BBF) are plotted versus metallicity, with $M / L_{\mathrm{V}}$ is in solar units. The metallicity of ellipticals is from the $\mathrm{Mg}_{2}-[\mathrm{Fe} / \mathrm{H}]$ relation of Buzzoni et al. (1992). Also shown is the $M / L_{\mathrm{V}}$ ratio of simple stellar population models for a 15 Gyr age, and the indicated IMF slope (Buzzoni, 1989, 1994).

Salpeter IMF $(x=1.35)$ a break at $[\mathrm{Fe} / \mathrm{H}]=-0.4$ appears that is tantalizingly close in size and position to the observed break. Why the steeper IMF generates the break? Before giving the solution I would like to put on the stage another piece of evidence. Fig. 3 displays the bolometric $M / L$ of Buzzoni's models $(x=1.35)$, showing that a sizable break is present also in bolometric light. However, the models of Worthey (1993) with the same IMF show instead an opposite break, with $M / L_{\mathrm{Bol}}$ decreasing with increasing metallicity.

To understand the effect one can spit $M / L_{\mathrm{V}}$ into the product $M / L_{\mathrm{Bol}}$. $L_{\mathrm{Bol}} / L_{\mathrm{V}}$. The term $L_{\mathrm{Bol}} / L_{\mathrm{V}}$ increases with increasing metallicity, which helps producing a tilt in the FP. However, in the metallicity range spanned by ellipticals in Fig. 2 this ratio increases by only $\sim 20-30 \%$ in Buzzoni's models, while $M / L_{\mathrm{Bol}}$ increases by $\sim 60 \%$ (for $x=1.35$ ). Therefore, the trend with metallicity is dominated by the decrease of $L_{\mathrm{Bol}}$, rather than by the shift to longer $\lambda \lambda$ of the SED. What matters is therefore the $Z$-dependence of $L_{\mathrm{Bol}}$. In turn, this is given by $L_{\mathrm{Bol}} \simeq 1.3 \times$ $10^{11} F_{\mathrm{T}}\left(M_{\mathrm{TO}}\right) b(t)$ (Renzini and Buzzoni, 1986; Renzini, 1994b, see their eq. 1.8), where $M_{\mathrm{TO}}(Z, t)$ is the mass of stars at the MS turnoff, $F_{\mathrm{T}}$ the amount of nuclear fuel burnt by evolving stars in the post-MS stages (in $\left.M_{\odot}\right)$, and $b(t)$ - the evolutionary flux of the population - is the rate at which stars leave the MS (in $\mathrm{yr}^{-1}$ ). In low mass stars $F_{\mathrm{T}}$ is almost independent of metallicity, but not so the evolutionary flux $b(t)=\psi\left(M_{\mathrm{TO}}\right) \dot{M}_{\mathrm{TO}}$, where $\psi$ is the IMF. Thus, what matters is the $Z$ dependence of $M_{\text {TO }}$, since $\dot{M}_{\text {TO }}$ is also fairly insensitive to $Z$. As $Z$ increases so does the metal contribution 


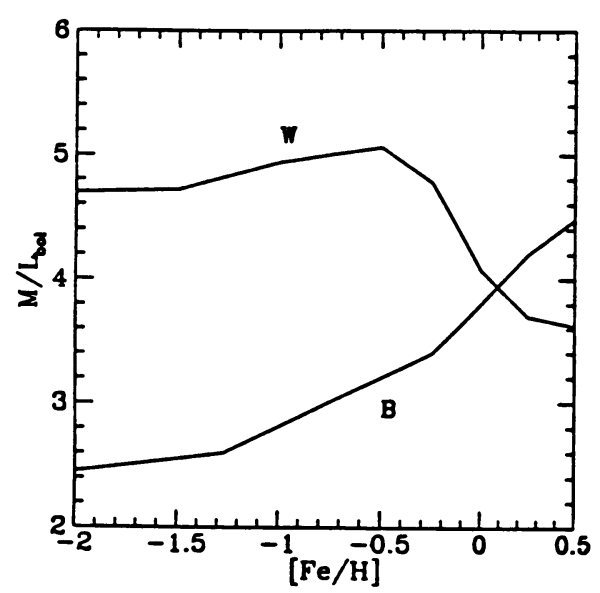

Figure 3. The bolometric mass to light ratio as a fuction of $[\mathrm{Fe} / \mathrm{H}]$ for the population models of Buzzoni (1989) and Worthey (1993), labelled "B" and "W", respectively. B models refer to and age of $15 \mathrm{Gyr}, \mathrm{W}$ models to $17 \mathrm{Gyr}$. Both sets of models are for $x=1.35$, but $\Delta Y / \Delta Z=1$ in B models, 2.5 in $\mathrm{W}$ models.

to opacity, stars are fainter and then take longer to exhaust hydrogen at their center. Thus, for given age $M_{\text {TO }}$ increases with increasing $\mathrm{Z}$, and this effect is further amplified in $L_{\mathrm{Bol}}$ as $L_{\mathrm{Bol}} \propto b(t) \propto M_{\mathrm{TO}}^{1+x}$. Thus, the larger $x$ the more pronounced the break in $M / L$ (see Fig. 2). Note that as far as the run of $M / L$ with $Z$ is concerned what matters is the IMF slope $x$ in a very restricted mass range just around $M_{\text {TO }}$. But while $M_{\text {TO }}$ increases for increasing $Z$, it instead decreases for increasing $Y$. For given mass, the higher helium the brighter the star and the shorter its lifetime. Thus, if $\Delta Y / \Delta Z \neq 0$ the turnoff mass may increase with $Z$ or decrease, depending on the actual $\Delta Y / \Delta Z$ (see Fig. 1 in Renzini, 1993), and the effect is further amplified in $b(t)$. This finally explains the divergence between the two sets of models in Fig. 3: simply one used $\Delta Y / \Delta Z=1$, the other $\Delta Y / \Delta Z=2.5$. It is nice to unravel a connection between helium and the FP ${ }^{1}$.

In conclusion, the tilt of the FP could be due to a mere metallicity effect provided two conditions are at once satisfied: 1 ) The IMF is rather steep around the MS turnoff ( $x \gtrsim 1.5)$, and 2) the helium enrichment is low $(\Delta Y / \Delta Z \lesssim 1)$. We don't know if the former condition is verified. If the result reported in $\S 2$ is correct $(\Delta Y / \Delta Z \simeq 3)$, then less than $\sim 1 / 3$ of the FP tilt is due to a metallicity effect on stellar evolution, and the residual $\sim 2 / 3$ should be due to either a dark matter systematic variation along the FP, or to a strong metallicity effect on star formation (the IMF). Yet another

\footnotetext{
${ }^{1}$ In this connection, it is instructive to briefly comment on the stellar model dependence of the $M / L(Z)$ relation. Using analytic expressions for $M_{\mathrm{TO}}(Z, t)$ brewed from stellar models using very old opacities (Greggio and Renzini, 1990) I once found $b(t)-$ and then $M / L_{\mathrm{Bol}}$ - almost independent of metallicity. This is at variance with Buzzoni's models, and the reason is that Buzzoni used stellar models based on newer opacities that are appreciably higher at high $Z$, and give a steeper $M_{\text {TO }}(Z)$ relation (see Fig. 2 in Iben and Renzini, 1984). It is amusing to realize that there is also a connection between opacity tables and the tilt of the FP!
} 
possibility will be briefly mentioned in the next section. The $M / L$ of the two metal rich clusters discussed in $\S 2$ is not yet available, but it would be very interesting to see what it is.

\section{Bounds on Elliptical Galaxy Ages: Discussion and Conclusions}

In this section I collect and comment a series of evidences that appear to support the old notion of an early formation for the bulk of stars in elliptical galaxies, with perhaps more than $\sim 90 \%$ being already in place at $z \gtrsim 2$.

- The tight color- $\sigma$ relation for Virgo and Coma ellipticals requires indeed a galaxy to galaxy age dispersion $\lesssim 15 \%$, or a formation epoch beyond $z \sim 2$, for $\gtrsim 90 \%$ of the stars in these galaxies (Bower et al. , 1992). Later additions should not exceed the residual $10 \%$.

- Precisely the same limits are independently imposed by the tightness of the FP, again for Virgo and Coma ellipticals (RC).

- Ellipticals in the $z=0.37$ cluster A370 appear to follow a $\mathrm{Mg}_{2}-\sigma$ relation that is shifted by an amount that requires the bulk of stars to have formed at $z \gtrsim 2$ (Bender, 1994, private communication).

- Clusters at high redshift $(z \sim 1)$ appear to contain a population of bright red galaxies, whose colors are consistent with merely passive evolution since $z \gtrsim 2$ (Aragon-Salamanca et al., 1993).

- The emitting region of QSO's at $z \gtrsim 3$ appears to be enriched in metals well above solar, suggestive of a very fast metal enrichment in the nuclear regions of galactic spheroids (Hamann and Ferland, 1993; Elston et al., 1994). If both the nuclei and haloes of spheroids form fast (most halo globulars were indeed in place $\sim 15$ Gyr ago), then why not the whole thing?

As correctly emphasized by Bower et al. for their own result, some of the points above do not strictly require an early formation. Formation might have occurred later as well, but if so the homogeneity of cluster ellipticals requires a very precise synchronization of formation epochs, or equivalently very similar star formation histories. A random formation, with galaxy to galaxy differences in the age for the bulk of stars larger than $\sim 15 \%$ would indeed result in fatter color- $\sigma$ and FP relations than is observed for Virgo and Coma ellipticals. However, all five evidences are naturally accounted for in the frame of the old scenario in which (most) spheroids form very early (see e.g., the models of Arimoto and Yoshii, 1987). Moreover, the tight correlation of metallicity with the depth of the galactic potential well (as exemplified by the color- $\sigma$ relation) favors dissipative collapse/merging for the formation process, as opposed to dissipationless merging.

At odds with the five arguments above appears to be the interpretation of the $\mathrm{H}_{\beta}-\mathrm{Mg}_{2}$ diagram for a sample of elliptical galaxies presented by Faber at this meeting, in which galaxies appear to follow an age rather 
than a metallicity sequence. While $\mathrm{Mg}_{2}$-strong galaxies are definitely $\sim 15$ Gyr old, $\mathrm{Mg}_{2}$-weak galaxies would perhaps be as young as $\sim 3 \mathrm{Gyr}^{2}$ (see also Worthey et al., 1992). Since $\mathrm{Mg}_{2}$ correlates with $\sigma$, i.e., mass and luminosity (the Faber-Jackson relation), this implies a tight correlation of formation epoch with mass, massive galaxies being older, lighter galaxies younger. This seems to go the other way around compared to a simple minded picture of hierarchical clustering, but - at least formally - is not necessarily in contradiction with the bounds set by the color- $\sigma$ and FP relations. If indeed the $\mathrm{Mg}_{2}$ sequence is driven by age instead of metallicity, then younger galaxies are in fact bluer and brighter, thus fulfilling also the color- $\sigma$ relation and offering an interpretation in terms of age of the tilt of the FP (younger populations have lower $M / L$ ). But again a very tight connection needs to be maintained over $\sim 1$ Hubble time between galaxy mass and formation epoch of the stellar component, a rather contrived situation.

It is worth stressing a fundamental difference between the first four of the five points above on the one hand, and the interpretation of the $\mathrm{H}_{\beta}-\mathrm{Mg}_{2}$ diagram on the other. Minimal use of population synthesis models is made in the former ones. Actually, only very robust predictions are involved, such as the time derivatives of colors and luminosity. On the contrary, theoretical $\mathrm{H}_{\beta}$ and $\mathrm{Mg}_{2}$ indeces are much more model dependent, being affected by large huge number of (some very uncertain) model ingredients. This is why, for the time being, I tend to assign a lower weight to arguments based on the $\mathrm{H}_{\beta}-\mathrm{Mg}_{2}$ diagram. Presently available observations are insufficient to disentangle between the two options of an age or a metallicity sequence, while the ambiguities of the population synthesis models may remain for a long time. A solution to the dilemma may perhaps come by looking at how the color $\sigma$ and FP relations evolve with redshift. If ellipticals form an age sequence, with the youngest ones as young as a few Gyr, then these objects should diasppear at redshifts as low as $\sim 0.3$ or so, with brighter and brigher objects disappearing at prograssively higher redshifts. Sufficiently complete luminosity functions of cluster ellipticals are required to test this possibility, which looks to be feasible at least with $10 \mathrm{~m}$ class telescopes.

\section{Appendix: An X-Ray Screening of the Standard Chemical Model}

It is worth reporting at this meeting that $\mathrm{X}$-ray observations with $B B X R T$, $R O S A T$, and $A S C A$ are consistently indicating a very low iron abundance $(\sim 1 / 3$ solar) for the X-ray gas within elliptical galaxies (see for example Awaki et al., 1994, and references therein). The composition of gas flows in ellipticals should reflect the average stellar abundances, selectively enriched

\footnotetext{
${ }^{2}$ Note however that using other models and other data Buzzoni et al. (1994) find galaxies along a metallicity sequence in their $\mathrm{H}_{\beta}-\mathrm{Mg}_{2}$ diagram.
} 
in iron by ongoing SNIa explosions. This implies an iron abundance from $\sim 2$ to several times solar, depending on the adopted SNIa rate in today ellipticals, and therefore there appears to be a discrepancy by perhaps as much as a factor of $\sim 10$ between expected and observed iron abundances. Preliminary analyses of $A S C A$ data for clusters of galaxies also suggest a $\mathrm{Ne} / \mathrm{Fe}$ ratio several times solar for the intracluster medium (Mushotzky, 1994). Together, the two results seem to exclude any significant role of Type Ia supernovae in manufacting iron in ellipticals, now as well as at earlier times. Perhaps there may be a problem with the interpretation of the ironL X-ray lines. But if not, X-ray data imply that the standard model for the galactic chemical evolution - with most $\alpha$-elements from SNII's and most iron from SNIa's - may not work at all for cluster ellipticals.

\section{References}

Aragon-Salamanca, A., Ellis, R.S., Couch, W.J. and Carter, D., 1993, M. N. R. A. S. 262, 764

Awaki, H., et al. , 1994, PASJ46, L65

Arimoto, N. and Yoshii, Y., 1987, A. \& A.173, 23

Bender R., Burstein D. and Faber S.M., 1992, Ap. J. 399, 462 (BBF)

Bower, R.G., Lucey, J.R. and Ellis, R.S., 1992, M. N. R. A. S. 254, 613

Buzzoni, A., 1989, Ap. J. Suppl. 71, 817

Buzzoni, 1994, Ap. J. , in press

Buzzoni, A., Carrasco, L., Salsa, M. and Recillas-Cruz, E., 1994, A. J. , in press

Buzzoni A., Gariboldi G., Mantegazza, L., 1992, A. J. , 103, 1814

Crane, P., et al. , 1993, A. J. 106, 1371

Djorgovski S. and Davis, M., 1987, Ap. J. 313, 59

Djorgovski S. and Santiago B.X., 1993, Structure, Dynamics and Chemical Evolution of Elliptical Galaxies, eds. Danziger I.J. et al. , Garching, ESO, p. 59

Dressler A., et al. , 1987, Ap. J. 313, 42

Elston, R., Thompson, K.L. and Hill, G.J., 1994, Nature 367, 250

Genzel, R., Hollenbach, D. and Townes, C.H., 1994, Rep. Prog. Phys. 57, 417

Greggio, L. and Renzini, A., 1990, Ap. J. 364, 35

Hamann, F. and Ferland, G., 1993, Ap. J. 418, 11

Holzman, J.A., et al. , 1993, A. J. 106, 1826

Iben, I. Jr., 1968, Nature 220, 143

Iben, I. Jr. and Renzini, A., 1984, Phys. Rep. 105, 329

Larson, R.B., 1975, M. N.R. A. S. 173, 671

Larson, R.B., 1990, P. A. S. P. 102, 709

Lee, Y.-W., 1992, A. J. 104, 1780

McWilliam, A. and Rich, R.M., 1994, Ap. J. Suppl. 91, 749

Mushotzky, R., 1994, in Clusters of Galaxies, ed. F. Durret. Gyf sur Yvette: Editions Frontières, in press

Ortolani S., Renzini, A., Marconi, G., Gilmozzi, R., Barbuy B. and Bica, E., 1994, in prep.

Paczyński, B. et al. , 1994, A. J. 107, 2060

Pryor C. and Meylan G., 1993, ASP Conf. Ser. 50, 357

Renzini, A., 1993, in Galactic Bulges, eds. H. Dejonghe and H.J. Habing, Dordrecht, Kluwer, p. 151

Renzini, A., 1994a, A. \& A.285, L5

Renzini, A., 1994b, Galaxy Formation, eds. J. Silk and N. Vittorio, Amsterdam: North 
Holland, in press

Renzini, A. and Buzzoni, A., 1986, Spectral Evolution of Galaxies, eds. C. Chiosi and A. Renzin, Dordrecht: Reidel, p. 135

Renzini, A. and Greggio, L., 1990, Bulges of Galaxies, eds. B.J. Jarvis and D.M. Terndrup, Garching: ESO, p. 47

Renzini A. and Ciotti, L., 1993, Ap. J. 416, L49 (RC)

Terndrup, D.M., 1988, A. J. 96, 884

Worthey G., 1992, Thesis, UCSC

Worthey G., Faber S.M. and Gonzales J.J., 1992, Ap. J. 398, 69

OSTRIKER: How much merging of different stellar populations to make an elliptical is permitted without destroying the thinness of the fundamental plane?

RENZINI: As much as can leave an age dispersion less than $\sim 15 \%$ for the bulk of the resulting stellar populations, for each luminosity of the resulting galaxies. To set more quantitative limits numerical simulations should be performed.

CARRASCO: The $M / L$ ratio is essentially constant with luminosity for galaxies in the Virgo and Coma clusters, when one goes to infrared light, as we can see from the data published by Recillas-Cruz et al. in the last few years.

RENZINI: To my recollection, Djorgovski and Santiago have used Recillas-Cruz et al. IR photometry to reach the conclusion that also $M / L_{\mathrm{K}}$ scales as $\sim L^{1 / 5}$. However I did not appreciate that there was such a serious disagreement between the two groups. It is definitely of prime importance to settle the question.

WHITE: 1) I think the lack of scatter in the $\mathrm{Mg}-\sigma$ relation and the fundamental plane shows that different ellipticals of the same luminosity have similar histories but not that they are old. 2) It is very dangerous to compare populations of different objects at different redshifts because we have no strong reason to believe that these populations correspond. Thus while all cluster galaxies at $z=0.5$ are probably still cluster members today, the reverse is almost certainly not true for many, perhaps for most present cluster members. Similarly, while most E galaxies seen at $z \sim 1$ may still be E's today (although some may have grown disks), most present day galaxies may have been pairs or small groups of disk objects at $z \sim 1$. 3) The models I showed in my own talk suggest that these two points may not be incompatible with the data you mention. The ellipticals were formed entirely by mergers in these models, yet they showed little scatter in the luminosity-color and luminosity-stellar $M / L$ relations. Furthermore, they show red ellipticals with little scatter in parameter relations at all redshifts; however, these relations do not evolve from redshift to redshift as they would for a fixed set of galaxies.

RENZINI: 1) I agree, the alternative to old age is indeed what Bower et al. call the synchronization in formation process. Absolute dating requires more than just color or luminosity derivatives. But the brightest ellipticals have so high $\mathrm{Mg}_{2}$ that existing population models indicate an age $\sim 15$ Gyr. 2) We certainly like to see how the population of cluster, group, and field galaxies evolve with lookback time, i.e., we like to have all apples and all oranges to play with. Then I agree that the comparison requires grain of salt. 3) I am impressed by your models. I would like to see them in detail, especially concerning the age distributions of stars in different galaxies. It is time for numerical simulations of structure formation to give detailed histories of what is then called a present day elliptical.

FABER: I would like to make a rather provoking comment. It might turn out that we two are focussing on apples + oranges. My sample yesterday was mostly galaxies in the field or weak clusters, plus several in Virgo. You today have focussed on 
two clusters, one of them very extreme (Coma). We actually have only one point of direct conflict, namely Virgo. It seems it might pay to take a really close look at what's going on in Virgo.

RENZINI: Admittedly, most of the evidence reported above refers to cluster ellipticals, and especially to those in Virgo and Coma. We know that field ellipticals may not be so homogeneous, and perhaps those in other clusters too. We clearly need more data for other field and cluster ellipticals, of quality comparable to that of the Virgo+Coma sample. I used this as being the best sample presently available. Also, note that Coma follows the same color- $\sigma$ and FP relations of Virgo (see Bower et al. 1992; BBF).

TAYLER: In your discussion of the metallicity of the cluster gas are you not making two implicit assumptions that all the gas has been through galaxies and that star formation occurred throughout the whole mass of the galaxies?

RENZINI: No. When dealing with elemental ratios there is no need to make that assumption. On the other hand, from the absolute abundances there is strong evidence that galactic winds have been diluted with pristine material, that was never incorporated into galaxies (stars).

NORRIS: Concerning the two globular clusters for which you have determined ages, how can you be sure that they are not members of the disk globular cluster population and that what you have measured is really the age of the disk rather than of the bulge?

RENZINI: The two clusters in Baade's Window are physically inside the bulge. It seems to me more prudent to call them "bulge clusters". Also, one of them has a radial velocity of $\sim 140 \mathrm{~km} \mathrm{~s}^{-1}$.

MINNITI: I have recently measured the He abundance in 5 different bulge fields. The result is $Y=0.28 \pm 0.02$.

FERGUSON: There are two cycle 2 HST programs aimed at detecting the evolution with redshift of the UV rising branch, both with FOS. Unfortunately, the spectra turned out to be seriously affected by red scattered light for $\lambda L_{\mathrm{T}} 2000$. Howver, S. Pescarelle has recently applied appropriate corrections to take out this effect, finding no residual UV flux in a $\mathrm{z} \approx 0.5$ weak radiogalaxy. Some UV rising branch remains, instead in $\mathrm{z} L_{\mathrm{T}} 0.2$ radiogalaxies, and one would conclude that the UV rising branch fades away somewhere between $\mathrm{z} \approx 0.2$ and 0.5 , as predicted if the UV rising branch is due to hot HB stars. Needless to emphasize that this is a very preliminary result that depends entirely on our ability of subtracting the rest scattered light in FOS spectra. 